\title{
AC 2008-37: USING THE FOURIER THEOREM TO TEST THE SOLUTION OF A DIFFERENTIAL EQUATION IN THE LAB
}

Josue Libii, Indiana University-Purdue University-Fort Wayne 


\title{
Using the Fourier Theorem to Test the Solution of a Differential Equation in the Laboratory
}

\begin{abstract}
This paper presents work conceived and implemented to test the validity of a secondorder differential equation that is commonly used to represent the oscillation of a springmass system. We designed a load cell and used it with computer data-acquisition equipment to collect data that give the position of the suspended mass versus time. We analyzed the collected data using the Fourier theorem and compared our experimental results to those obtained by solving the differential equation analytically. Students found it remarkable that the analysis of collected data demonstrated in a convincing manner that the analytical solution represented the motion of a mass suspended from a spring accurately.
\end{abstract}

\section{Introduction}

One way to bring excitement in the use of mathematics in the engineering classroom is to show that it can be used to model physical reality accurately. This paper presents work conceived and implemented to test the extent to which an ordinary differential equation and its solution are valid for use in actual applications. The equation chosen is commonly used in mathematics, physics, and engineering courses.

We consider the ordinary differential equation given by

$\ddot{x}+\omega_{n}^{2} x=0$,

with the following initial conditions

$x(t=0)=x_{0}$

$\dot{x}(t=0)=v_{0}$

where $\mathrm{x}$ is a function of time, the dots indicate derivatives of $\mathrm{x}$ with respect to time, and $\omega_{n}^{2}$ is a quantity that is independent of time. 
This equation has been used to model a variety of physical phenomena. For example, it can represent the motion of a mass, $\mathrm{m}$, suspended onto a linear spring of stiffness $\mathrm{k}$, when losses due to friction are negligible. In that case, the natural frequency and natural period of the motion of the mass are given, respectively by

$\omega_{n}=\sqrt{\frac{k}{m}}$ and $\tau=\frac{2 \pi}{\omega_{n}}=2 \pi \sqrt{\frac{m}{k}}$.

The general solution to Eq. (1) subject to the initial conditions shown in Eq. (2) is

$x(t)=x_{0} \cos \omega_{n} t+\frac{v_{0}}{\omega_{n}} \sin \omega_{n} t$.

Equations (1), (2), and (3) are encountered in many undergraduate courses in mathematics, physics, and engineering ${ }^{1,2}$.

This paper discusses an experiment that was designed to test the validity of Eq. (3) in the laboratory. The experiment demonstrates the extent to which Eq.(3) is a reliable model for the oscillations of a mass that is suspended to a linear spring in a laboratory setting.

The remainder of this paper is organized in the following manner: first, the Fourier theorem is stated and its use in the present context is discussed. Then, the design of the experiment is presented. Next, experimental data are presented and analyzed using the Fourier theorem to generate the Fourier coefficients. Finally, these experimental coefficients are compared to those derived from the solution of the differential equation itself.

\section{The Fourier theorem and its use}

If $x(t)$ is a periodic function with period $\tau$, its Fourier series representation is given by

$$
x(t)=\frac{a_{0}}{2}+\left(a_{1} \cos \omega t+b_{1} \sin \omega t\right)+\left(a_{2} \cos 2 \omega t+b_{2} \sin 2 \omega t\right)+\left(a_{3} \cos 3 \omega t+b_{3} \sin 3 \omega t\right)+\ldots .
$$

In compact notation, this expression takes the form

$$
x(t)=\frac{a_{0}}{2}+\sum_{n=1}^{\infty}\left(a_{n} \cos n \omega t+b_{n} \sin n \omega t\right)
$$

where $\omega \equiv \frac{2 \pi}{\tau}$ is the fundamental frequency and $a_{0}, a_{1}, a_{2}, a_{3}, a_{2}, a_{3}, \ldots, b_{1}, b_{2}, b_{3}, \ldots$ are constant coefficients obtainable from the evaluation of the following integrals 
$a_{0} \equiv \frac{2}{\tau} \int_{0}^{\tau} x(t) d t$

$a_{n} \equiv \frac{2}{\tau} \int_{0}^{\tau} x(t) \cos (n \omega t) d t$

$b_{n} \equiv \frac{2}{\tau} \int_{0}^{\tau} x(t) \sin (n \omega t) d t$

In practice, the function $\mathrm{x}(\mathrm{t})$ is not always available in the form of a mathematical expression. Indeed, in may cases, $\mathrm{x}(\mathrm{t})$ is obtained from experimental data, or as a result of numerical computation. In such cases, $\mathrm{x}(\mathrm{t})$ is available as a set of $\mathrm{N}$ pairs of numbers $\left[t_{i}, x\left(t_{i}\right)\right], i=1,2,3, \ldots N$, where each pair indicates a specific time, $\mathrm{t}_{\mathrm{i}}$, at which the corresponding functional value $\mathrm{x}\left(\mathrm{t}_{\mathrm{i}}\right)$ of $\mathrm{x}(\mathrm{t})$ is known. Then, to obtain the Fourier coefficients, $a_{0}, a_{n}$, and $b_{n}$, one can use the following numerical approximations of the integrals shown in Eq. (5) ${ }^{1-3}$.

$$
\begin{aligned}
& a_{0} \approx \frac{2}{N} \sum_{i=1}^{N} x\left(t_{i}\right) \\
& a_{n} \approx \frac{2}{N} \sum_{i=1}^{N} x\left(t_{i}\right) \cos \left(\frac{2 n \pi t_{i}}{\tau}\right) \\
& b_{n} \approx \frac{2}{N} \sum_{i=1}^{N} x\left(t_{i}\right) \sin \left(\frac{2 n \pi t_{i}}{\tau}\right)
\end{aligned}
$$

Issues relating to whether or not the Fourier series will converge to $x(t)$ at every value of $t$ in the relevant interval have been examined and settled by mathematicians: it is known that the function $x(t)$ must satisfy Dirichlet conditions ${ }^{2,3}$. In particular, if $x(t)$ is continuous within the period, then, its Fourier series will converge to $x(t)$ at every point. Also, if $x(t)$ has a discontinuity at some time $t$, the Fourier series will converge to the average, in other words to the midpoint, of the discontinuity.

In the experiment described below, data were collected from the oscillation of a mass that was suspended onto the end of a linear spring. The data themselves consisted of a set of instantaneous positions of the oscillating mass relative to its position of rest and the corresponding times. These data are used in Eq. (6) to determine approximations for the Fourier coefficients $a_{0}, a_{n}$, and $b_{n}$. These are used in the Fourier theorem to construct a mathematical expression that corresponds to the collected data. The resulting function is then compared to the solution of the governing differential equation that was shown in 
Eq. (3) in order to examine the extent to which collected data agree with the analytical solution.

\section{The experiment}

A sphere of mass $m=1.584 \mathrm{~kg}$ is attached to the lower end of a linear spring that was tested and found to be linear. The stiffness of the spring was determined to be $\mathrm{k}=117 \mathrm{~N} / \mathrm{m}$ and its mass was negligible compared to that of the sphere. The upper end of the spring was hooked onto a sensor similar to the Whittmore-Petrenko proving ring that has been tested and demonstrated to be a linear transducer ${ }^{4}$. This spring-mass assembly is in the vertical plane. The proving ring is connected to a computer data acquisition system that makes it possible to record the position of the sphere as a function of time ${ }^{5}$.

In order to have very simple and repeatable initial conditions, the sphere was set into motion by giving it an initial displacement $\mathrm{x}=\mathrm{x}_{0}$ downwards and holding the sphere stationary in that position for five seconds before releasing it from rest, that is, without any initial velocity. The value of $\mathrm{x}_{0}$ was recorded. However, in order to make it easy to compare data from successive trials, the recorded displacements are normalized by diving each of them by $\mathrm{x}_{0}$. A sample set of collected data is shown in Table 1, where the time, $\mathrm{t}$, is in seconds and the tabulated position of the sphere is $\frac{x}{x_{0}}$, a dimensionless ratio.

In Table 1, the first column gives the time, in seconds, at which the position of the center of the sphere had the coordinate value shown in column two. Column three is a continuation of column 1 and column four is continuation of column two. Position data are dimensionless because they were normalized by diving each entry by the amplitude of oscillation of the sphere during the tested cycle. That amplitude was taken to be the initial displacement of the sphere. The time data were not normalized, however. 
Table1. A sample of collected data

\begin{tabular}{||r||r||r||l||}
\hline $\begin{array}{l}\text { Time } \\
\text { (s) }\end{array}$ & \multicolumn{1}{l||l}{$\begin{array}{l}\text { Position } \\
(-)\end{array}$} & $\begin{array}{l}\text { Time } \\
\text { (s) }\end{array}$ & \multicolumn{1}{l||}{$\begin{array}{l}\text { Position } \\
(-)\end{array}$} \\
\hline \hline 0 & -0.90847 & 0.37 & 1 \\
\hline 0.017 & -0.87254 & 0.395 & 0.984692 \\
\hline 0.034 & -0.85317 & 0.415 & 0.930334 \\
\hline 0.051 & -0.80631 & 0.435 & 0.84911 \\
\hline 0.068 & -0.75289 & 0.455 & 0.753515 \\
\hline 0.094 & -0.61262 & 0.481 & 0.593877 \\
\hline 0.114 & -0.48079 & 0.501 & 0.442987 \\
\hline 0.134 & -0.3527 & 0.522 & 0.284599 \\
\hline 0.155 & -0.17432 & 0.542 & 0.124649 \\
\hline 0.182 & 0.042799 & 0.563 & -0.04061 \\
\hline 0.203 & 0.196189 & 0.588 & -0.25555 \\
\hline 0.223 & 0.36676 & 0.608 & -0.41643 \\
\hline 0.243 & 0.523586 & 0.629 & -0.55514 \\
\hline 0.263 & 0.664792 & 0.649 & -0.6776 \\
\hline 0.289 & 0.804749 & 0.67 & -0.79225 \\
\hline 0.309 & 0.892534 & 0.695 & -0.8388 \\
\hline 0.329 & 0.962824 & 0.712 & -0.89097 \\
\hline 0.349 & 0.999063 & 0.729 & -0.91034 \\
\hline \hline
\end{tabular}

\section{Analysis of experimental data}

A study of the data shown in Table 1 indicates that they correspond approximately to one complete cycle of oscillation that has a natural period of $0.729 \mathrm{~s}$. This compares favorably with the analytical period of $0.730 \mathrm{~s}$, which is obtainable from Eq. (2a) after letting $\mathrm{k}=117 \mathrm{~N} / \mathrm{m}$ and $\mathrm{m}=1.584 \mathrm{~kg}$. The discrepancy between the two is $0.14 \%$.

The data shown in Table 1 will be analyzed further using the Fourier theorem. They can be processed automatically to generate the corresponding Fourier coefficients using software such as Mathematica, Matlab, or Maple. However, one may also choose to use a manual approach in class, which is particularly useful when students are working with the Fourier theorem for the first time. In this approach, they compute the coefficients using hand-held calculators, or a spreadsheet such as Excel. The instructional advantage of this approach is that these tools require students to enter the data and the formulas that 
are needed for the determination of the coefficients manually. Such hands-on applications allow for familiarity with and, hopefully, a deeper understanding of the meaning and use of the Fourier theorem. Table 2, shown below, displays the detailed computations that were carried out in order to obtain the Fourier coefficients for the case of $n=1$ [1]. Similar tables were generated for higher values of $n$. In the exercise done in class, this process was carried out for $n=0$ through $n=6$. The resulting coefficients are shown in Table 3(a). Naturally, applying the Fourier theorem to the solution shown in Eq.(3) with the initial conditions used in this experiment yields a single nonzero Fourier coefficient, as shown in Table 3(b).

Table 2. Detailed computations of the Fourier coefficients for $n=1$.

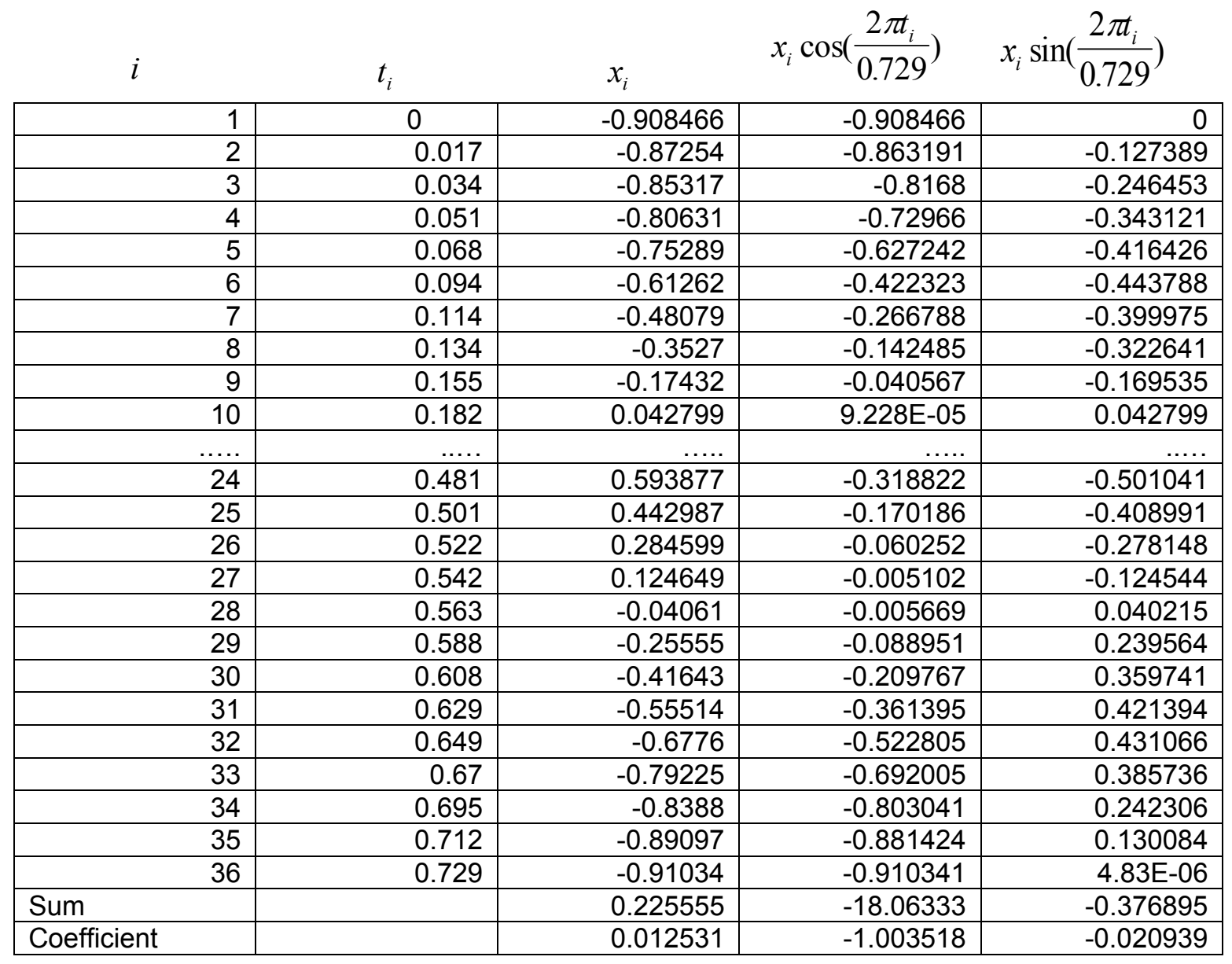


Table 3(a). Experimental Fourier coefficients

\begin{tabular}{|l|r|r|r|}
\hline $\mathrm{n}$ & \multicolumn{1}{|c|}{$a_{n}$} & \multicolumn{1}{c|}{$b_{n}$} & $c_{n}$ \\
\hline 0 & 0.0548641 & 0 & 0.0548641 \\
\hline 1 & -0.957223 & -0.0100514 & 1.0037366 \\
\hline 2 & -0.00173248 & 0.0034438 & 0.097333 \\
\hline 3 & 0.00308254 & 0.00475898 & 0.0877436 \\
\hline 4 & 0.00142011 & 0.00109271 & 0.0767017 \\
\hline 5 & 0.00257423 & -0.000909756 & 0.0763974 \\
\hline 6 & -0.000637367 & 0.000949299 & 0.0554893 \\
\hline
\end{tabular}

Table 3(b). Fourier coefficients from theory

\begin{tabular}{|l|r|r|r|}
\hline $\mathrm{n}$ & $a_{n}$ & $b_{n}$ & \multicolumn{1}{|c|}{$c_{n}$} \\
\hline 0 & 0 & 0 & 0 \\
\hline 1 & -1 & 0 & 1 \\
\hline 2 & 0 & 0 & 0 \\
\hline 3 & 0 & 0 & 0 \\
\hline 4 & 0 & 0 & 0 \\
\hline 5 & 0 & 0 & 0 \\
\hline 6 & 0 & 0 & 0 \\
\hline
\end{tabular}

The series corresponding to Tables 3(a) and 3(b) are represented in the Fourier domain in Fig. 1 and in the time domain in Fig. 2. It can be seen from these figures and from Table 3 that the second term in the Fourier series corresponds quite closely to the solution that was determined by solving the differential equation analytically. This suggests that, perhaps, terms with higher frequencies represent a combination of experimental errors and the effects of losses in the system. Such losses might be due to dry friction between the spring hook and the supports and to hysteretic friction in the spring itself. Losses due to air resistance were found to be very small over one single cycle of oscillation. However, the losses due to air resistance have a cumulative effect over time. Indeed, they eventually damp out the motion completely after approximately eight hundred cycles. Assuming that this explanation is sufficient to account for the presence of higher order terms, the experiment yielded data that demonstrated the validity of the model shown in Equation (1) and supported its applicability in the laboratory setting. 

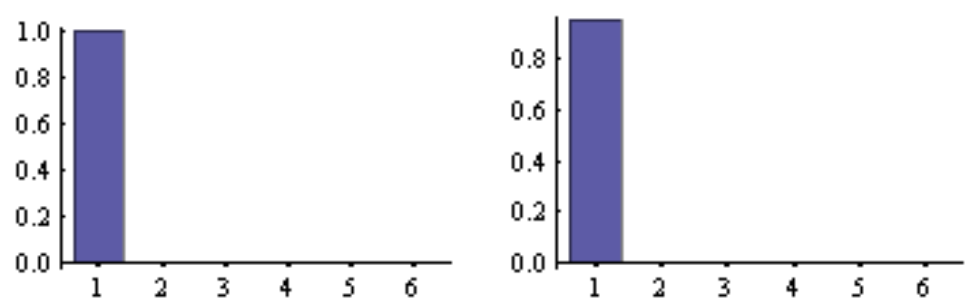

Figure 1. Fourier spectra from theory (left) and from data (right)
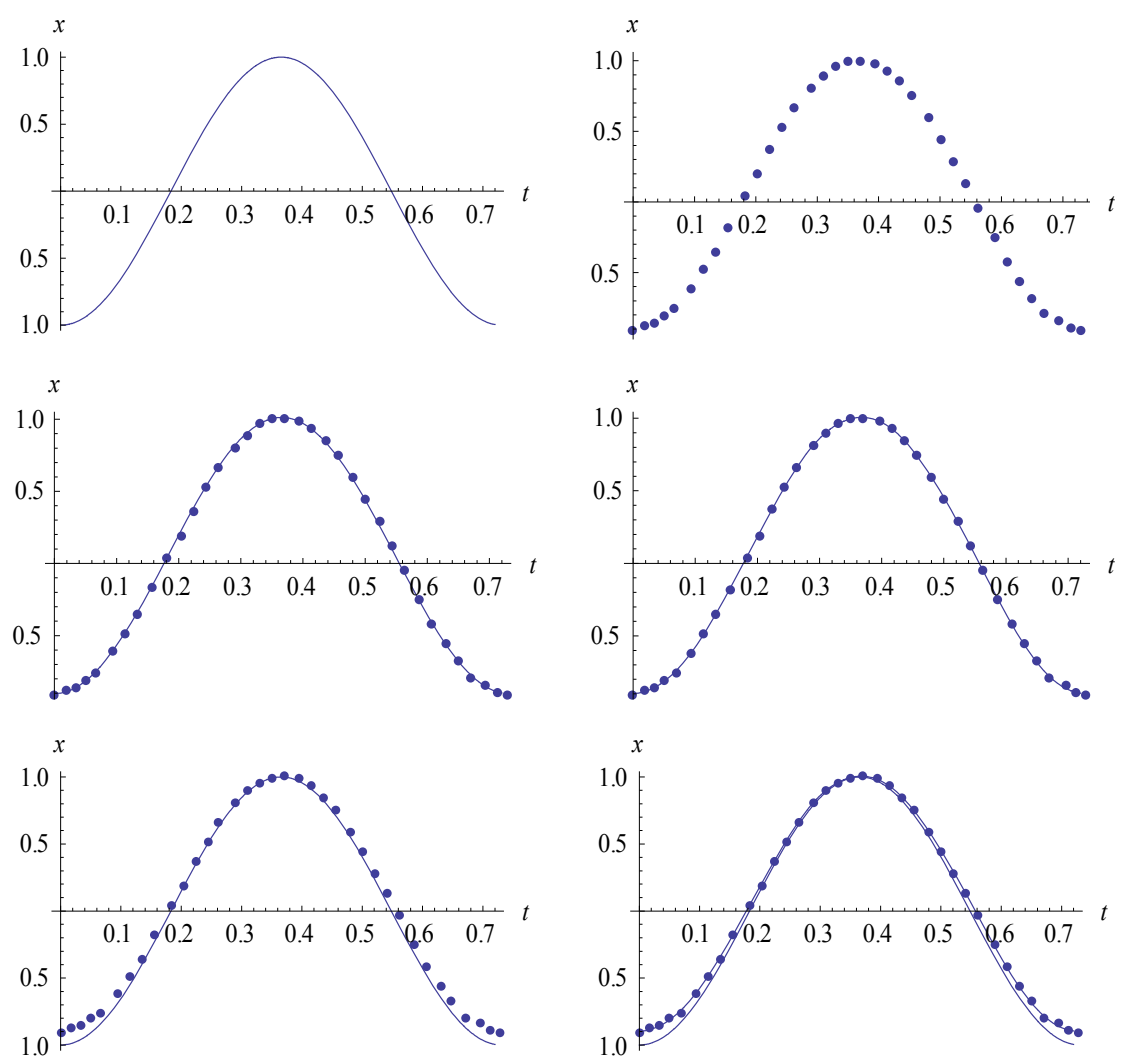

Fig. 2. Plots compare the analytical solution to both raw data and to their Fourier series. First row: plots of the analytical solution (left) and of the raw data (right);

Second row: data and their Fourier series $(n=1)$; data and their Fourier series $(n=6)$; Third row: the solution and the Fourier series $(n=1)$; solution and Fourier series $(n=6)$. 


\section{Discussion of Fig. 2}

Fig. 2 is a graphical illustration of the results of the experiment. It is presented in three rows, each containing two images. The first row shows a plot of the analytical solution obtained from the differential equation (plot on the left) and a plot of the raw data obtained from the experiment (plot on the right). It was presumed that one could capture the mathematical behavior represented by the experimental data by applying the Fourier theorem to them. When it was completed, that application resulted in a Fourier series that had infinitely many terms.

As a practical matter, one needed to truncate the series by deciding how many terms to keep. To this end, the second row of Fig. 2 compares the raw data to their Fourier series: the figure on the left shows a plot of the raw data on which a truncated Fourier series consisting of one trigonometric term is superposed; and the figure on the right shows similar plots, except that the truncated series with one term has been replaced with one consisting of six trigonometric terms. The discrepancies between raw data the series are less than $0.1 \%$ using one term, and less than $1 \%$ using six terms. This almost exact agreement allows one to use the Fourier series with one term as an accurate representation of the mathematical behavior of the experimental data.

The third row compares the analytical solution to the Fourier series that was derived from experimental data: to this effect, the figure on the left shows a plot of the solution of the differential equation on which a truncated Fourier series consisting of one trigonometric term is superposed; and the figure on the right shows similar plots, except that the truncated series with one term has been replaced with one consisting of six trigonometric terms. Discrepancies between the solution to the differential equation and the Fourier series representation of the data vary during a given oscillation. They are largest at the beginning and end of the cycle (5\%) and smallest in the middle of it $(0.01 \%)$. It is speculated that such discrepancies may be due to imprecise initial conditions. In this case, it is possible that the ball was released with small amounts of rotation and, perhaps, with a small initial velocity.

\section{Conclusions}

It is very common to model the free oscillation of a mass that is suspended to the end of a linear spring using an ordinary differential equation of second order with constant coefficients, the solution to which consists of circular functions. An experiment was designed and carried out to test the validity of this model for practical applications. The 
chief purpose was to demonstrate the extent to which the mathematical solution is a reliable model for actual oscillations of a mass in the laboratory.

Instantaneous positions of a mass suspended onto a spring were collected versus time using a sensor that was connected to computer data-acquisition equipment; these data were analyzed using the Fourier theorem and compared to what was expected from analysis. Comparisons demonstrated that, when comparison is limited to one single period of motion, the differential equation being investigated represented the motion of the mass accurately. Indeed, the first non-constant term in the Fourier series that was obtained from experimental data and the analytical solution to the differential equation itself differed by less than $2.5 \%$, on average. However, when a large number of periods was taken into account, the amplitude of motion decreased with time due to the cumulative effect of air resistance. Then, the mathematical model being investigated ceased to be valid 5 .

\section{References}

1. Rao, S.S., Mechanical Vibrations (4 ${ }^{\text {th }}$ edn), Reading, Massachusetts: Addison-Wesley, 2004, pp.129152.

2. Proakis, J.G., and Manolakis, D. G., Introduction to digital signal processing, Macmillan Publishing Company, New York, New York, 1988, pp. 219-299.

3. Churchill, R.V., Fourier series and boundary value problems, second edition, McGraw-Hill Book Company, New York, New York, 1969, pp. 77-112.

4. Josué Njock Libii, Design, analysis and testing of a force sensor for use in teaching and research, World Transactions on Engineering and Technology Education, Vol.5, No. 1, 2006, pp. 175-178.

5. Josué Njock Libii, Demonstration of viscous damping in the undergraduate laboratory, American Journal of Physics, 68 (2), 2000, pp. 195 -198. 